\title{
Quimiogenómica y Quimiogenética: Definiciones, técnicas y aplicaciones en el ámbito farmacéutico
}

\author{
Chemogenomics and Chemogenetics: \\ Definitions, techniques and applications \\ in the pharmaceutical area
}

\author{
Javier Arroyo-Rodríguez¹, Felipe González-Young², \\ Raquel Hernández-Vega ${ }^{3}$, Cristian Lizano-Quirós ${ }^{4}$, \\ Josué Quesada-Murillo ${ }^{5}$, Juan José Mora-Román ${ }^{6}$
}

Arroyo-Rodríguez, J; González-Young, F; Hernández-Vega, R; Lizano-Quirós, C; Quesada-Murillo, J; Mora-Román, JJ. Quimiogenómica y Quimiogenética: Definiciones, técnicas y aplicaciones en el ámbito farmacéutico. Tecnología en Marcha. Vol. 33-1. Enero-Marzo 2020. Pág 3-16.

doi) https://doi.org/10.18845/tm.v33i1.5017

1 Estudiante de Licenciatura en Farmacia, Facultad de Farmacia, Universidad de Costa Rica, San José, Costa Rica. Correo electrónico: javier.arroyo@ucr.ac.cr. (iD) https://orcid.org/0000-0002-1357-6944

2 Estudiante de Licenciatura en Farmacia, Facultad de Farmacia, Universidad de Costa Rica, San José, Costa Rica. Correo electrónico: luis.gonzalezyoung@ucr.ac.cr. (iD) https://orcid.org/0000-0001-7677-7965

3 Estudiante de Licenciatura en Farmacia, Facultad de Farmacia, Universidad de Costa Rica, San José, Costa Rica. Correo electrónico: raquel.hernandezvega@ucr.ac.cr. (iD) https://orcid.org/0000-0002-1532-1814

4 Estudiante de Licenciatura en Farmacia, Facultad de Farmacia, Universidad de Costa Rica, San José, Costa Rica. Correo electrónico: cristhian.lizano@ucr.ac.cr. (iD) https://orcid.org/0000-0001-6114-1366

5 Estudiante de Licenciatura en Farmacia, Facultad de Farmacia, Universidad de Costa Rica, San José, Costa Rica. Correo electrónico: josue.quesadamurillo@ucr.ac.cr. (iD https://orcid.org/0000-0002-8560-0966

6 Máster en Bioquímica, Departamento de Farmacia Industrial, Facultad de Farmacia, Universidad de Costa Rica, San José, Costa Rica. Correo electrónico: juanjose.moraroman@ucr.ac.cr. 


\title{
Palabras clave
}

Biotecnología; Quimiogenética; Quimiogenómica; Farmacia; DREADDs.

\section{Resumen}

En los años 80, tomó fuerza la ciencia dirigida al estudio del genoma humano, cuyo objetivo era mejorar la salud humana a través de la identificación de genes. Este estudio permitió el surgimiento de ciencias, entre ellas la Quimiogenómica y la Quimiogenética. La Quimiogenómica es un área interdisciplinaria que busca la sistematización del genoma al identificar, analizar, expandir y predecir las interacciones de los ligandos con las proteínas por medio de métodos in vitro e in silico. Por otro lado, la Quimiogenética se refiere al estudio de las características genéticas de un paciente para optimizar la Farmacoterapia y predecir la eficacia, los efectos secundarios y la dosificación de fármacos selectos. El conocimiento de las interacciones entre moléculas y proteínas específicas ayudaron al desarrollo de nuevas herramientas biotecnológicas y a la identificación de novedosas dianas farmacológicas que pudieran conducir a conocimientos más específicos. Algunas aplicaciones en estudio en el ámbito farmacéutico son los receptores diseñados exclusivamente activados por fármacos diseñados (DREADDs, por sus siglas en inglés). Con ellos se pretende que no se activen ante ligandos endógenos, sino en presencia de sintéticos. Actualmente existen muchas investigaciones en los cuales se estudian DREADDs para la identificación de neuronas y de vías de señalización involucradas en patologías como el miedo, la ansiedad, la depresión, la adicción y la obesidad.

\section{Keywords}

Biotechnology; Pharmacogenomics; Pharmacogenetics; Pharmacy; DREADDs.

\begin{abstract}
In the decade of 1980, the science that studied human genome rose strongly, with the purpose of improving human health by identifying genes. These studies allowed the origin of other sciences like Chemogenomics and Chemogenetics. Chemogenomics is an interdisciplinary area that looks for the genome systematization by identifying, analyzing, expanding and predicting the interactions of ligands and proteins using the in vitro and in silico methods. In the other hand, Chemogenetics is the study of the genetic characteristics of a patient to optimize the pharmaceutical therapy and predict the efficacy, the secondary effects and the dosage of certain drugs. The knowledge of molecules and proteins interaction helped to develop new biotechnological tools and to identify novel drug targets that could conduct to more specific knowledge. One of the applications in Pharmacy are the designer receptors exclusively activated by designer drugs (DREADDs). The DREADDs may not allow the activation by the endogenous ligands, but in presence of endogenous ones. Actually, there are lots of investigations in which DREADDs are being studied for the identification of neurons and signalization routes involved in pathologies such as fear, anxiety, depression, addiction, and obesity.
\end{abstract}




\section{Introducción}

Genómica es el campo que se encarga de los estudios estructurales y funcionales del genoma [1] (información genética total del organismo), y la función e interacción del ADN en este, así como los factores ambientales o no genéticos como el estilo de vida personal [2]. El genoma contiene secuencias, denominados genes, con información necesaria para generar moléculas con función biológica [3]. Dicha información se almacena en cromosomas.

Por otra parte, la Genética corresponde al estudio de los genes individuales, junto con su participación en una enfermedad y su modo de herencia [2]. En conjunto con la Genómica, buscan explicar las causas de diversas enfermedades y así, mejorar las intervenciones y los resultados terapéuticos. Este conocimiento ha sido notable en el desarrollo práctico de la Farmacoterapia y desempeñan un papel importante en el diagnóstico, la prevención y el tratamiento de las enfermedades [2] [3].

Por ende, la presente revisión bibliográfica tiene como propósito dar a conocer técnicas quimiogenómicas y quimiogenéticas, así como su importancia y su aplicación en el descubrimiento y en el desarrollo de fármacos aplicados en las terapias individualizadas de distintas patologías.

\section{Antecedentes}

La ciencia dirigida al estudio del genoma tomó fuerza a mediados de la década de 1980, en la que se discutió la posibilidad de secuenciar el genoma humano. En 1986, Charles DeLisi del Departamento de Energía de Estados Unidos financió el comienzo del proyecto dirigido a mapear y secuenciar dicho genoma. Como complemento, en 1988 se dio un financiamiento mayor por parte del gobierno y el apoyo de James Watson con la formación del Instituto de Salud Nacional ( $\mathrm{NIH}$, por sus siglas en inglés) [4]. El objetivo del Proyecto Genoma Humano $(\mathrm{PGH})$ era mejorar la salud de la humanidad a través de su caracterización y la identificación de los genes. Gracias a la ayuda internacional y el esfuerzo cooperativo, se logró realizar en menor tiempo al esperado [3] [4] [5] [6].

Del PGH resultó una cantidad inmensa de información (tres mil millones de nucleótidos componen el genoma humano), la cual requería ser tratada y analizada de una forma óptima. De esta necesidad nació la Bioinformática. Su finalidad fue generar bases de datos y herramientas para un estudio funcional comprensivo, y mecanismos para producir programas computarizados que pudieran ser compartidos y exportados [7] [8] [9].

Una de las ciencias generadas como consecuencia de la aparición de esta tecnología fue la Genómica. Consiste en la identificación, la clasificación de miembros de familias de genes (que se presume tienen cierta homología) y la determinación de su función. La misma se extendió, no sólo a la secuenciación del genoma, sino que promovió la aparición de la Genómica Funcional, con distintas ramas, entre ellas la Proteómica [8]. La misma se refiere al estudio de la función y la composición de todas las proteínas expresadas en una muestra dada [9] [10].

Continuando con los avances en el campo, se desarrolló la Quimiogenética, también llamada Farmacogenética o Farmacosíntesis. De manera general, permite utilizar las características genéticas de un paciente para optimizar la Farmacoterapia y predecir la eficacia, los efectos secundarios y la dosificación de fármacos selectos [2]. El término Quimiogenética fue el primero en acuñarse y se separó de la connotación directa de la Farmacología y la Genética, aunque puede estar relacionado a ellas [11]. 
Posteriormente, en 1996, investigadores de la empresa Glaxo Wellcome introdujeron el término Quimiogenómica [12]. Se trata de una disciplina que combina las últimas herramientas de Genómica y Química, y se enfoca en las dianas y el descubrimiento de fármacos. De esta forma, trata de determinar los posibles compuestos capaces de interactuar con una diana terapéutica [13] [14].

Es así como por primera vez, gracias a la investigación dirigida hacia familias de genes blanco, se discutió el concepto de la sistematización del descubrimiento de fármacos basándose en el análisis de genes blanco como receptores acoplados a proteínas G (GPCRs, por sus siglas en inglés), canales iónicos o proteasas que habían sido explorados de manera exitosa hasta el momento [12] [14]. Esto se originó al recalcar las ventajas de poseer enfoques basados en sistemas de combinación de avances en clonación, expresión, automatización de genes, química combinatoria y Bioinformática [12].

El principio básico en el cual los investigadores se basaron fue que ligandos similares se deberían unir a blancos similares, pudiendo aplicar y transferir los conocimientos adquiridos de proyectos anteriores a otros nuevos. En 2001, la farmacéutica Vertex introdujo la implementación de plataformas de Quimiogenómica para el descubrimiento de inhibidores de receptores quinasa. Asimismo, Novartis describió los principios del diseño de una biblioteca de Quimiogenómica para el blanco de familias biogénicas de GPCRs relacionados a aminas. A partir de esto, muchas publicaciones e investigaciones se han realizado para entender las interacciones de blanco-ligando y crear una matriz llamada relación estructura-actividad (SAR, por sus siglas en inglés) [12] [15].

\section{Quimiogenómica}

Es un área interdisciplinaria que busca la sistematización del genoma al identificar, analizar, expandir y predecir las interacciones de los ligandos con las proteínas, por medio de métodos in vitro e in silico. De esta área nace la Quimiogenómica Computacional, la cual toma esta información y la integra en bases de datos, específicamente en modelos predictivos destinados al descubrimiento, y a la validación de moléculas y enfermedades específicas como blancos terapéuticos [12].

La Quimiogenómica difiere del enfoque farmacéutico convencional, porque vincula directamente proyecciones de bibliotecas extensas. Estas bibliotecas contienen millones de compuestos similares, los cuales pueden ser sintetizados utilizando ADN recombinante o combinaciones químicas [16] a metodologías de genómicas modernas. Para lograr esto, es necesario tener acceso a nuevas "bibliotecas de compuestos sintéticos [17]". En el descubrimiento de fármacos basados en ella se proyectan grandes colecciones de productos químicos para la identificación de dianas y compuestos biológicamente activos [14] [18].

Los compuestos biológicamente activos que se descubren bajo esta metodología se conocen como terapias dirigidas, pues se unen y modulan dianas moleculares específicas. La idea de esta estrategia es acelerar el ritmo del proceso de descubrimiento de fármacos [18]. Al ser una oportunidad para convertir rápidamente los estudios fenotípicos de enfermedades en el descubrimiento de drogas enfocados en las dianas farmacológicas encontradas, es posible reposicionar las ya existentes, hacer predicciones de su toxicología y descubrir nuevas modalidades farmacológicas. Además, asegura que un fármaco pueda tener la eficacia terapéutica deseada [19].

Entre algunos métodos computacionales que se emplean con frecuencia en la Quimiogenómica se encuentran el cribado virtual (CV) y la búsqueda computacional de blancos moleculares (target fishing en inglés) [17]. Ambas técnicas permiten la predicción de efectos adversos de un compuesto, la detección de la polifarmacología de una droga (interacción de un fármaco con varias dianas favorables) y el reposicionamiento de un fármaco [17] [20]. 
De manera general, el cribado virtual filtra una serie de compuestos de bases de datos moleculares para seleccionar un conjunto de moléculas que tienen más probabilidad de unirse a un objetivo específico [20]. Existen muchos métodos como el de proteína-ligando. El mismo trata de obtener predicciones rápidas y exactas de la conformación 3D que adopta un ligando al interaccionar con una proteína, así como la fuerza de unión para evaluar funciones de afinidad. Una de las limitaciones de este método es la falla en la predicción de la toxicidad y de la actividad, al estar limitada por el acceso a recursos computacionales [17] [21].

Por otra parte, la búsqueda computacional de blancos moleculares se entiende como la identificación de uno o varios blancos moleculares más probables de la molécula en estudio [17] [20]. Esto se logra por medio de varios métodos computacionales clasificados en cuatro grupos: búsqueda de similitud química, minería de datos/aprendizaje automático, acoplamiento de paneles y análisis de espectros de bioactividad. También, se han propuesto otros basados en la estructura proteica [20].

Dos métodos adicionales en la Quimiogenómica son los quimioinformáticos y la Quimiogenómica in silico. La Quimioinformática se emplea para analizar cuantitativamente la diversidad química, la visualización del espacio químico, y el contenido y la diversidad de núcleos base [13]. Por otra parte, la Quimiogenómica in silico busca crear una matriz bidimensional en la cual las dianas se encuentran en columnas y los ligandos en filas [13] [14]. Los valores numéricos son constantes de enlace o efectos funcionales [13].

Para predecir las afinidades, la Quimiogenómica se basa en el principio de la similitud [13]. Este concepto es abstracto y trata de cuantificar el parecido que hay entre dos estructuras químicas [17]. El principio de similitud, el cual es la base de muchos métodos empleados, establece que moléculas similares podrían unirse al mismo blanco y por ende, tener bioactividades similares [13] [20]. Esta va a depender de la representación molecular y la métrica para cuantificar el grado de acercamiento entre ellos [13] [17].

\section{Quimiogenética}

Inicialmente, el término Quimiogenética había sido acuñado para describir los efectos observados por mutaciones en enzimas-sustratos específicos. Ahora, se utiliza para describir el proceso en que macromoléculas metabólicas como ácidos híbridos, quinasas, y una variedad de enzimas y GPCRs pueden ser sintetizados para interactuar con pequeñas moléculas que no han sido reconocidas hasta el momento [22]. También se define mediante el acrónimo de DREADDs (por sus siglas en inglés), que significa receptores diseñados exclusivamente activados por fármacos diseñados [23].

Es una técnica que permite el control remoto de las poblaciones de células y circuitos neuronales, por medio de inyecciones sistémicas o micro infusión de un ligando activador. Para ello, utiliza técnicas con receptores diseñados y ligandos biológicos inertes. Es poco invasiva y útil para realizar mapeos funcionales, manipulación específica de ciertos tipos de células y control de neuronas [24].

Un ejemplo son los GPCRs. Gracias a la Quimiogenética, estas proteínas pueden excitar, inhibir o modular las neuronas. Su potencial permite la unión a ligandos cognados (del mismo origen) relativamente inactivos. No obstante, existe un problema con respecto a la utilización de moléculas sintéticas, pues no generan una señal suficiente o que pueda ser usada como herramienta para el control remoto de la señalización neuronal [11]. 
En resumen, la Farmacogenética estudia el efecto del fármaco en un individuo según su variación genética, mientras que la Farmacogenómica trata de la Genética de las enfermedades para orientar el desarrollo de nuevas vías de tratamiento. Con frecuencia, suelen ser confundidos y usados como términos iguales. Sin embargo, ambos estudian la función de la célula por medio de pequeñas moléculas sintetizadas que sirven como ligandos moduladores [18]. El uso complementario de ambos puede guiar al desarrollo de fármacos más selectivos y a la comprensión de mejores sitios de acción de ciertas enfermedades.

\section{Importancia de los modelos para la evaluación de fármacos}

El proceso de descubrimiento de fármacos inicia con la identificación de compuestos que se unen a un blanco terapéutico o que muestran actividad biológica en un ensayo de tamizaje. Luego, se estudian aquellos compuestos con propiedades farmacéuticas atractivas que incluyan: baja toxicidad, solubilidad acuosa y otras propiedades farmacocinéticas requeridas [25].

Dentro de los requisitos solicitados para permitir la comercialización de un producto farmacéutico se encuentran los estudios clínicos controlados. Estos deben ser validados por Sistemas de Gestión de la Calidad, los cuales determinan que la molécula en estudio puede ser autorizada y comercializada posterior a la demostración de seguridad y de eficacia. No obstante, antes de esta etapa es necesario pasar por la de investigación preclínica [26].

Los estudios preclínicos se realizan en animales y modelos fisiológicos en el laboratorio. Los modelos in vivo posibilitan la aplicación de técnicas de análisis que por cuestiones éticas no pueden realizarse en seres humanos. Además, permite investigaciones que no se obtienen mediante técnicas in vitro, donde no pueden reproducir las interacciones propias de un sistema biológico completo [26] [27].

A través de los modelos animales es posible caracterizar la patología de la enfermedad, evaluar el mecanismo de acción de las drogas existentes, descubrir nuevos blancos terapéuticos y biomarcadores, establecer las relaciones farmacodinamia/farmacocinética, estimar los regímenes de dosis clínicos, y determinar los parámetros de seguridad y toxicidad, para salvaguardar la integridad de los voluntarios de las pruebas clínicas en sus distintas fases [26] [27].

Sin embargo, estos limitan la extrapolación de los resultados a los estudios en seres humanos. Al tratarse de sistemas biológicos distintos, existen diferencias de respuesta entre ellos. El problema parece ser la traducción inter especies para las reacciones adversas inesperadas y la eficacia de la sustancia de interés. Tal situación genera la mayoría de los retiros de drogas y fármacos en fase de ensayo clínico o incluso del mercado. Sin embargo, lo anterior podría reducirse mediante la mejora de los procesos de pruebas preclínicas, tomando en cuenta las tecnologías recientes [26] [27].

Por ello, uno de los principales retos del diseño de fármacos actualmente es la identificación de aquellos que interaccionen con dianas biológicas deseadas, pero que a la vez no sean blancos moleculares asociados a efectos adversos [17]. Se trata de un proceso complejo y costoso en el cual convergen diferentes áreas de conocimiento [25].

Si se conoce la base biológica de un proceso patológico a nivel molecular y se identifican las moléculas implicadas en el mismo, así como su interacción con otras rutas metabólicas, es posible encontrar un punto sobre el cual se pueda intervenir para su modificación. Este punto por lo general es una proteína. Por ello, justamente la secuenciación del genoma humano ha ampliado el campo referente al diseño de fármacos [13]. 
El conocimiento de las interacciones entre moléculas y proteínas específicas ayudan al desarrollo de novedosas herramientas biotecnológicas y a la identificación de nuevas dianas farmacológicas que puede conducir a conocimientos más específicos. Estos se puede lograr mediante cribado quimiogenómicas. Por medio de ellas, cada pequeña molécula de una biblioteca química se aplica a cada tipo de una biblioteca de células y se registran los fenotipos resultantes [28].

De esta manera, aunque el descubrimiento y el desarrollo de medicamentos se ha hecho durante muchos años usando únicamente métodos experimentales, el diseño de fármacos asistido por computadora (DIFAC) tiene cada vez más importancia en su investigación y su desarrollo. Se espera que este proceso se acelere gracias a los métodos computacionales para codificar con precisión modelos teóricos y para procesar grandes cantidades de información [25]. EI DIFAC forma parte de un esfuerzo multidisciplinario y está conformado por una serie de disciplinas científicas que abarcan Modelado Molecular, Quimioinformática, Química Teórica y Química Computacional [17].

Por lo anterior, el poder predictivo de los métodos basados en secuencias ha comenzado a atraer el interés en las últimas etapas de los estudios preclínicos. La identificación temprana de otras dianas donde podría interactuar el medicamento es crucial para el desarrollo de nuevos fármacos [14]. Sin embargo, la práctica incorrecta de usar métodos de cómputo contribuye a crear falsas expectativas de que pueden diseñar fármacos por sí solos. Se debe tener la claridad de que estos no generan de manera automatizada los medicamentos, sino que deben estar integrados con pruebas experimentales por medio de varios ciclos de optimización [17].

\section{Técnicas quimiogenómicas}

A continuación se muestran las técnicas comúnmente empleadas por la Quimiogenómica para el desarrollo de fármacos. Las mismas se dividen en aquellas basadas en el enfoque al sitio de unión de la diana y las basadas en el ligando.

Basadas en el enfoque al sitio de unión de la diana

\section{Enfoque basado en la secuencia}

Usualmente para buscar las similitudes en las dianas múltiples y únicas se utilizan herramientas básicas de búsqueda de alineamiento local (BLAST, por sus siglas en inglés) y análisis filogenéticos basados en la sustitución como las matrices de sustitución de bloques de aminoácidos (BLOSUM, por sus siglas en inglés) y las mutaciones puntuales aceptados (PAMs, por sus siglas en inglés). La Quimiogenómica también se puede utilizar para la identificación de dianas únicas y múltiples, optimizado la interacción del ligando con la diana. Al igual que la aproximación clásica, utiliza técnicas como descriptores fisicoquímicos y farmacóforos de aminoácidos, analizando la secuencia de cada uno y las posibles interacciones existentes entre ellos [20] [29].

\section{Enfoque basado en la estructura}

Se han desarrollado numerosas bases de datos encargadas de representar el sitio de unión como: Relibase, PDBLIG, PDBBind, sc-PDB, entre otros [30]. Algunos de los recursos aplicados con el fin de definir la estructura son la cocristalización (dos o más especies neutras contenidas en un mismo cristal que se mantienen unidas por interacciones no covalentes) [31] y la detección de cavidades. Una característica en común es la realización de estructuras 3D a los farmacóforos representativos, los cuales son utilizados para realizar comparaciones [20] [29]. 


\section{Basadas en el ligando}

\section{Reposicionamiento de la droga y ligando}

Los efectos que se presentan fuera de la diana pueden tener una ventaja, pues permiten el reposicionamiento del fármaco para indicaciones alternas. La modificación de compuestos es capaz de convertir un efecto secundario en uno principal [32]. Posibles nuevas dianas se obtienen a partir de cruces individuales para cada ligando. Para ello, se efectúa el acople de afinidades para un conjunto de compuestos, independientemente de la estructura de y la secuencia de la diana [33].

\section{Bibliotecas enfocadas en estructuras privilegiadas}

Algunas subestructuras se han encontrado en ligandos, de los cuales no tienen relación. Estas fueron llamadas estructuras privilegiadas, debido a su versatilidad [34]. Las mismas pueden unirse a muchos receptores. También se ha visto que cuando se sustituyen, dan lugar a ligandos potentes y muy selectivos. Esta técnica tiene la utilidad de que se puede escalar para predicciones con todos los GPCRs [29].

\section{Aplicación de la Quimiogenética en el descubrimiento y el desarrollo de fármacos}

Los GPCRs son los mayores objetivos farmacológicos en el descubrimiento de nuevos fármacos, debido a que participan en la modulación de muchos procesos patológicos y fisiológicos en los seres humanos [35]. Por esta razón, el hallazgo y el desarrollo de nuevos fármacos van destinados hacia este tipo de receptores [36]. El diseño de DREADDs con diferentes perfiles farmacológicos es una herramienta con potencial para descubrir nuevos fármacos relacionados con GPCRs [11]. El principio fundamental es modificar receptores celulares, de tal manera que sean incapaces de responder al ligando endógeno. No obstante, se diseñan para que puedan ser activados por moléculas inertes como ligandos sintéticos [22].

A diferencia del mecanismo de acción de los moduladores alostéricos, dependientes de la presencia del neurotransmisor endógeno, el DREADD funciona independientemente del ligando endógeno. Su expresión en lugar del receptor muscarínico natural en sistemas biológicos resulta en un fenotipo similar al que se aprecia en modelos animales que han sido privados de este receptor mediante técnicas "knock-out" [37]. Un modelo de ratón "knock-out" se fundamenta en la disrupción del marco de lectura de un gen, con la intención de bloquear la expresión del mismo en un organismo vivo. Los modelos murinos con estas características son útiles para el estudio del rol biológico que poseen ciertos alelos genéticos en la síntesis de proteínas específicas [38].

La administración del ligando sustituto «sintético» activa el DREADD, permitiendo la identificación de respuestas celulares y fisiológicas asociadas con la activación del receptor mutante. Este enfoque imita las respuestas que podrían esperarse si se desarrollara un fármaco selectivo para un determinado subtipo de receptor. Por ende, permite la evaluación sistemática de los posibles efectos farmacológicos que podrían esperarse como respuesta a su activación [22].

Uno de los requisitos para el uso del ligando sintético exógeno utilizado para activar el receptor diseñado es que sea farmacológicamente inerte en condiciones fisiológicas. Además, la modificación genética que se introduce en este debe ser capaz de incapacitar la respuesta del mismo en presencia del ligando endógeno, así como garantizar la ausencia de actividad [22]. 


\section{Desarrollo de DREADDs}

Originalmente se utilizaron técnicas de evolución molecular dirigida y un sistema de expresión basado en levaduras con el objetivo de diseñar un receptor muscarínico $\mathrm{M}_{3}$ que fuera capaz de responder al ligando exógeno N-óxido de clozapina (CNO, por sus siglas en inglés) y eliminar la señalización intrínseca relacionada con el ligando endógeno acetilcolina (ACh, por sus siglas en inglés). Se debe tener en cuenta que en condiciones normales el CNO carece de completa actividad a nivel del receptor muscarínico $\mathrm{M}_{3}$ de tipo salvaje. Por ello, su escogencia como ligando de partida para la creación del primer receptor mutante muscarínico de tipo DREADD no resultó intuitiva [39] [40] [41].

En contraste, la clozapina, molécula derivada del CNO, presenta en condiciones fisiológicas alta afinidad por el receptor muscarínico $\mathrm{M}_{3}$ de tipo salvaje. Debido a esta propiedad, se predijo que la introducción de pocas mutaciones en el código genético del receptor muscarínico sería suficiente para que el CNO adquiriera la afinidad necesaria para funcionar como agonista del receptor mutante diseñado, pero manteniendo su nula afinidad sobre el receptor muscarínico de tipo salvaje. Además, las propiedades farmacocinéticas del CNO eran ideales, porque presentaban muy buena biodisponibilidad tanto en ratones como en humanos [42] [43].

Como línea celular para el diseño de los DREADD se utilizó de manera original la levadura Saccharomyces cerevisiae. La línea celular específica escogida poseía la característica de que el GPCR nativo de este microorganismo fue removido, pero la subunidad G $\alpha$ natural fue preservada y modificada mediante técnicas de Ingeniería Genética, de tal manera que pudiera interactuar con GPCRs de origen humano. Si la línea celular con estas características era funcional, la activación del receptor humano acoplado a la subunidad alfa de levadura desencadenaría una cascada de señalización, activada en condiciones naturales por feromonas, que culminaría en una promoción del crecimiento fúngico en un medio selecto. El experimento original implicó además la introducción del gen HIS3, cuya transcripción es promovida por la activación de la cascada de señalización mencionada anteriormente. La utilidad de introducir este gen subyace en que subyacía en que permitiría que la levadura modificada genéticamente proliferara en medios de cultivo deficientes en histidina [43].

Una vez que se tuvo definida la línea celular de levadura con un receptor muscarínico funcional de origen humano y el gen HIS3, se procedió a evaluar combinaciones de posibles modificaciones en la estructura conformacional de la proteína que constituía el receptor para determinar la existencia de afinidad con el ligando CNO. Para lograrlo, se generó una biblioteca de receptores mutantes análogos al de origen humano, la cual se insertó en la línea celular de levadura [39]. Para generar estos análogos, se utilizaron técnicas de mutagénesis aleatoria basadas en la reacción en cadena de la polimerasa (PCR, por sus siglas en inglés). Dicha técnica permite la introducción de mutaciones dentro de las secuencias de una plantilla predeterminada de ADN con la finalidad de obtener variantes genéticas de proteínas que sean de interés investigativo [44].

Esta estrategia permitió descubrir que únicamente se necesitaban dos mutaciones puntuales específicas en residuos de la proteína del receptor (cambio de tirosina por cisteína en el tercer dominio transmembrana o Y3.33C, y sustitución de alanina por glicina en el quinto dominio transmembrana o A5.46G), para lograr que respondiera al ligando sintético exógeno CNO, que perdiera la sensibilidad por la acetilcolina y que se obtuvieran niveles bajos de actividad basal constitutiva. El receptor diseñado recibió el nombre de hM3Dq (receptor muscarínico humano de diseño acoplado a proteína $G_{q}$ ). Debido a que Y3.33 y A5.46 son residuos conservados en la familia de proteínas de receptores muscarínicos, con esta misma tecnología se lograron diseñar versiones mutantes del resto de subtipos de receptores muscarínicos acoplados a proteína $G_{a}$ $\left(\mathrm{M}_{1}\right.$ y $\left.\mathrm{M}_{5}\right)$ [39]. 


\section{Nuevas variantes de DREADDs}

La técnica original de mutagénesis dirigida permitió esclarecer cuáles residuos de aminoácidos específicos en los dominios transmembrana del receptor muscarínicos eran los que aportaban la afinidad por la acetilcolina en condiciones fisiológicas naturales, y cómo las mutaciones puntuales de estos generaban selectividad exclusiva por una droga sintética e impropia de sistemas biológicos. De esta forma, se crearon DREADDs acoplados a proteínas $\mathrm{G}_{i}$ al introducir mutaciones en Y3.33 y A5.46 de receptores muscarínicos humanos $M_{2}$ y $M_{4}$ [45].

Los receptores metabotrópicos muscarínicos acoplados a proteína $G_{i}$ están involucrados en la activación de canales rectificadores de entrada de potasio (GIRKs, por sus siglas en inglés) a través del dímero formado por las subunidades $\beta \gamma$ de la proteína G [46]. Este dímero favorece la apertura del canal GIRK y el eflujo de cationes potasio $\left(\mathrm{K}^{+}\right)$, resultando en un proceso de hiperpolarización de la membrana celular [47]. Esta propiedad de los receptores muscarínicos del subtipo $\mathrm{M}_{2} / \mathrm{M}_{4}$ ha sido emulada en células de riñón de embrión humano (HEK-239) mediante la expresión del DREADD de diseño M4. Por tal razón, esta tecnología sería de utilidad para "silenciar" neuronas o disminuir su actividad en modelos in vivo e in vitro orientados al estudio de circuitos neurales y su modulación de funciones fisiológicas [39] [48].

Si bien originalmente se diseñaron receptores con esta tecnología que únicamente podían acoplarse a proteínas $G_{q}$ y $G_{i}$, existe una variante quimérica del receptor sintético $M_{3}$ que incorpora características funcionales del receptor $\beta$-adrenérgico presente en eritrocitos de pavo. Específicamente, este receptor quimérico fue creado al sustituir los dominios intracelulares 2 y 3 del receptor muscarínico de diseño con secuencias del adrenérgico. A diferencia de las variantes originales de los muscarínicos, este DREADD adrenérgico puede provocar cascadas de señalización, porque se acopla a una proteína $G_{s}$. Se diseñó originalmente para el estudio del metabolismo de la glucosa en ratones transgénicos que expresaban este DREADD de manera selectiva en células $\beta$ pancreáticas. La activación del mismo por el ligando exógeno CNO provocó estados de hiperglucemia, mimetizando la respuesta fisiológica pancreática que se observa ante una descarga adrenérgica de catecolaminas. La única desventaja fue la presentación de bajos niveles de actividad constitutiva [41] [49].

\section{Expresión en sistemas biológicos}

Cuando estos receptores de diseño son activados mediante la unión de CNO pueden desencadenar la cascada de señalización natural acoplada al tipo específico de proteína G con que hayan sido construidos. Se ha demostrado que este mecanismo sucede cuando se inserta el receptor sintético $\mathrm{M}_{3}$ en células de riñón de embrión humano (HEK-239) cultivadas in vitro, en fibroblastos de embrión de ratón que expresan el DREADD mediante técnicas de transfección in vitro [49] y en poblaciones neuronales in vivo e in vitro [39] [50].

También se ha demostrado su funcionamiento en astrocitos, hepatocitos y células $\beta$ pancreáticas en un contexto in vivo [41] [51]. En cada una de estas poblaciones celulares, los efectos fisiológicos que se derivan de la transducción de señales a través del DREADD son diferentes, como consecuencia de la heterogeneidad y la amplia gama de tejidos que pueden ser utilizados para expresarlo [48].

Debido a que estos DREADDs especializados se pueden expresar en poblaciones de células diana o células asociadas a una patología o enfermedad específica, si uno de estos receptores de diseño se ha insertado en una población celular particular y es eficaz para revertir el fenotipo de una determinada enfermedad estudiada, esa población de células podría ser analizada mediante técnicas biotecnológicas (secuenciación de ARN) para encontrar las dianas moleculares de interés [11]. Posteriormente, se pueden expresar dichas dianas en cultivos celulares y sintetizar moléculas capaces de modularlas para reproducir la cascada 
de señalización desencadenada por el DREADD. Una vez que se ha identificado un posible candidato que sea capaz de replicar el comportamiento inducido por el receptor mutante, se puede evaluar la molécula en modelos animales [52].

Elución de la función de los receptores de opioides

Los opioides son un grupo de fármacos utilizados en el alivio del dolor, los cuales se unen y activan a sus receptores (acoplados a proteínas $\mathrm{G}$ ). Sus efectos están mediados principalmente por el receptor opioide mu (MOR, por sus siglas en inglés). Este tiene dos tipos de receptores altamente relacionados: delta (DOR, por sus siglas en inglés) y kappa (KOR, por sus siglas en inglés) [53].

Se ha encontrado que el estado de los receptores opioides mu de los ratones pueden encontrarse estables con el uso de nanocuerpos de alta resolución. Los mismos se derivan de la cadena pesada de los anticuerpos. Poseen propiedades superiores comparadas con anticuerpos convencionales como mayor solubilidad, menor inmunogenicidad y una habilidad única de unión con epítopos [54].

Usando receptores opioides KOR de alta resolución con modelamiento molecular y mutagénesis, la salvinorina B (metabolito inactivo de la salvinorina A, la cual es un potente agonista de los KOR) mostró una potencia agonista mejorada para el KOR mutante D138N. También se le aplicó nitrógeno para que interactuara como el resto de péptidos endógenos opioides. De esta forma, la salvinorina B pudo interaccionar con el KOR mutante D138N, el cual mostró una afinidad mejorada para salvinorina B y exhibió insensibilidad a todos los agonistas peptídicos opioides y agonistas no peptídicos que contenían nitrógeno. A partir de ahí se les denominó receptores opioides DREADD kappa (KORD, por sus siglas en inglés) [55], los cuales han sido utilizados para silenciar neuronas in vivo [56].

\section{Conclusiones}

El uso de métodos o técnicas que involucren un estudio interdisciplinario ha llevado al descubrimiento de nuevos compuestos con actividad biológica. La utilización de la Quimiogenética tiene un gran potencial en el ámbito de la investigación farmacéutica. Lo anterior se debe a que el conocimiento de las interacciones entre moléculas y proteínas específicas ayudan al desarrollo de nuevas herramientas biotecnológicas y a la identificación de novedosas dianas farmacológicas para conducir a conocimientos más específicos. Algunas aplicaciones en estudio en el ámbito farmacéutico son los DREADDs. Con ellos, se pretende que no se activen ante ligandos endógenos, sino en presencia de sintéticos. Actualmente existen muchas investigaciones en los cuales se estudian estos receptores para la identificación de neuronas y de vías de señalización involucrados en patologías como el miedo, la ansiedad, la depresión, la adicción y la obesidad. Por todo lo anterior, se espera un futuro muy promisorio en esta área en el corto y mediano plazo.

\section{Referencias}

[1] A. Cervantes Peredo, "Genómica, medicina y sociedad," Revista Médica del Hospital General de México, 66(4), 224-234, 2003.

[2] J. L. Jameson and P. Kopp, "Principios de genética humana," en Harrison. Principios de Medicina Interna, 19 ed., D. Kasper, A. Fauci, S. Hauser, D. Longo, J. L. Jameson, and J. Loscalzo, Ed. Nueva York: Mc Graw Hill Medical, 2016.

[3] I. Silva Zolezzi, "Genómica y medicina," Educación Química, 22(1), 15-27, 2011. 
[4] F. S. Collins, M. Morgan, and A. Patrinos, "The Human Genome Project: Lessons from Large-Scale Biology," Science, 300(5617), 286-290, 2003.

[5] E. S. Lander, L. M. Linton, B. Birren, C. Nusbaum, M. C. Zody, J. Baldwin et al, "Initial sequencing and analysis of the human genome," Nature, 409(6822), 860-921, 2001.

[6] J. C. Venter, M. D. Adams, E. W. Myers, P. W. Li, R. J. Mural, G. G. Sutton et al, "The sequence of the human genome," Science, 291(5507), 1304-1351, 2001.

[7] E. Barreto-Hernández and M. T. Reguero Reza, "Grupo de Bioinformática: En la frontera del análisis de datos moleculares," Revista Colombiana de Biotecnología, 2017: Conmemoración IBUN 30 años, 23-26, 2017.

[8] S. Wu-Pong and R. Shiang, "The Use of Bioinformatics and Chemogenomics in Drug Discovery," in Biopharmaceutical Drug Design and Development, 2 ed., S. Wu-Pong and Y. Rojanasakul, Ed. Berlin: Springer, 2008, pp. 31-45.

[9] A. Jara y J. J. Kopchick, "Proteómica: una aproximación integral," Anales de Pediatría, 78(3), 137-139, 2013.

[10] M. Tyers and M. Mann, "From genomics to proteomics," Nature, 422(6928), 193-197, 2003.

[11] S. M. Sternson and B. L. Roth, "Chemogenetic Tools to Interrogate Brain Functions," Annual Review of Neuroscience, 37, 387-407, 2014.

[12] E. Jacoby, Computational Chemogenomics, New Western United States: Taylor \& Francis Group, 2013.

[13] L. Delgado-Soler y J. Rubio Martínez, "Quimiogenómica: una nueva aproximación al diseño de fármacos," Farmespaña Industrial, 32, 73-75, 2009.

[14] C. J. Harris and A. P. Stevens, "Chemogenomics: structuring the drug discovery process to gene families," Drug Discovery Today, 11(19-20), 880-888, 2006.

[15] D. Gupta-Ostermann and J. Bajorath, "The 'Sar Matrix' method and its extensions for applications in medicinal chemistry and chemogenomics (version 2)," F1000 Research, 3(113), 2014.

[16] S. Ahn, A. W. Kahsai, B. Pani, Q. T. Wang, S. Zhao, A. L. Wall et al, "Allosteric "beta-blocker" isolated from a DNA-encoded small molecule library," Proceedings of the National Academy of Sciences of the United States of America, 114(7), 1708-1713, 2017.

[17] J. L. Medina-Franco, E. Fernández-de Gortari y J. J. Navaja, "Avances en el diseño de fármacos asistido por computadora," Educación Química, 26(3), 180-186, 2015.

[18] M. Bredel and E. Jacoby, "Chemogenomics: an emerging strategy for rapid target and drug discovery," Nature Reviews Genetics, 5(4), 262-275, 2004.

[19] L. H. Jones and M. E. Bunnage, "Applications of chemogenomic library screening in drug discovery," Nature Reviews Drug Discovery, 16(4), 285-296, 2017.

[20] A. Cereto-Massagué, M. J. Ojeda, C. Valls, M. Mulero, G. Pujadas, and S. Garcia-Vallve, "Tools for in silico target fishing," Methods, 71, 98-103, 2015.

[21] H. Pérez-Sánchez, G. Cano, J. García-Rodríguez y J. M. Cecilia, "Descubrimiento de fármacos basado en cribado virtual refinado con enfoques neuronales paralelos," Revista Internacional de Métodos Numéricos para Cálculo y Diseño en Ingeniería, 31(4), 207-211, 2015.

[22] D. J. Urban and B. L. Roth, "DREADDs (Designer Receptors Exclusively Activated by Designer Drugs): Chemogenetic Tools with Therapeutic Utility," Annual Review of Pharmacology and Toxicology, 55, 399-417, 2015.

[23] K. S. Smith, D. J. Bucci, B. W. Luikart, and S. V. Mahler, "DREADDS: Use and application in behavioral neuroscience," Behavioral Neuroscience, 130(2), 137-155, 2016.

[24] E. J. Campbell and N. J. Marchant, "The use of chemogenetics in behavioural neuroscience: receptor variants, targeting approaches and caveats," British Journal of Pharmacology, 175(7), 994-1003, 2018.

[25] F. Saldívar-González, F. D. Prieto-Martínez y J. L. Medina-Franco, "Descubrimiento y desarrollo de fármacos: un enfoque computacional," Educación Química, 28(1), 51-58, 2017.

[26] T. I. Ramos, "Contexto actual de los estudios preclínicos," Bionatura, 1(3), 103-105, 2016.

[27] P. McGonigle and B. Ruggeri, "Animal models of human disease: Challenges in enabling translation," Biochemical Pharmacology, 87(1), 162-171, 2014.

[28] A. Wuster and M. Madan Babu, "Chemogenomics and biotechnology," Trends in Biotechnology, 26(5), 252258, 2008.

[29] D. E. Gloriam, "Chemogenomics of allosteric binding sites in GPCRs," Drug Discovery Today: Technologies, 10(2), e307-e313, 2013. 
[30] B. Pirard, "Structure-Based Chemogenomics: Analysis of Protein Family Landscapes," Methods in Molecular Biology, 575, 281-296, 2009.

[31] Z. Gao, S. Rohani, J. Gong, and J. Wang, "Recent Developments in the Crystallization Process: Toward the Pharmaceutical Industry," Engineering, 3(3), 343-353, 2017.

[32] T. T. Ashburn and K. B. Thor, "Drug repositioning: identifying and developing new uses for existing drugs," Nature Reviews Drug Discovery, 3(8), 673-683, 2004.

[33] M. Bieler, R. Heilker, H. Köppen, and G. Schneider, "Assay Related Target Similarity (ARTS) - Chemogenomics Approach for Quantitative Comparison of Biological Targets," Journal of Chemical Information and Modeling, 51(8), 1897-1905, 2011.

[34] B. E. Evans, K. E. Rittle, M. G. Bock, R. M. DiPardo, R. M. Freidinger, W. L. Whitter et al, "Methods for Drug Discovery: Development of Potent, Selective, Orally Effective Cholecystokinin Antagonists," Journal of Medicinal Chemistry, 31(12), 2235-2246, 1988.

[35] H. M. Lee, P. M. Giguere, and B. L. Roth, "DREADDs: novel tools for drug discovery and development," Drug Discovery Today, 19(4), 469-473, 2014.

[36] J. A. Allen and B. L. Roth, "Strategies to Discover Unexpected Targets for Drugs Active at G Protein-Coupled Receptors, Annual Review of Pharmacology and Toxicology, 51, 117-144, 2011.

[37] B. L. Roth, "DREADDs for Neuroscientists," Neuron, 89(4), 683-694, 2016.

[38] B. Hall, A. Limaye, and A. B. Kulkarni, "Overview: Generation of Gene Knockout Mice," Current Protocols in Cell Biology, 44(1), 19.12.1-19.12.17, 2009.

[39] B. N. Armbruster, X. Li, M. H. Pausch, S. Herlitze, and B. L. Roth, "Evolving the lock to fit the key to create a family of G protein-coupled receptors potently activated by an inert ligand," Proceedings of the National Academy of Sciences of the United States of America, 104(12), 5163-5168, 2007.

[40] S. J. Bradley, A. B. Tobin, and R. Prihandoko, "The use of chemogenetic approaches to study the physiological roles of muscarinic acetylcholine receptors in the central nervous system," Neuropharmacology, 136(Part C), 421-426, 2018.

[41] J. M. Guettier, D. Gautam, M. Scarselli, I. Ruiz de Azua, J. H. Li, E. Rosemond et al, "A chemical-genetic approach to study G protein regulation of B cell function in vivo," Proceedings of the National Academy of Sciences of the United States of America, 106(45), 19197-19202, 2009.

[42] J. L. Gomez, J. Bonaventura, W. Lesniak, W. B. Mathews, P. Sysa-Shah, L. A. Rodriguez et al, "Chemogenetics revealed: DREADD occupancy and activation via converted clozapine," Science, 357(6350), 503-507, 2017.

[43] Y. Pei, S. Dong, and B. L. Roth, "Generation of Designer Receptors Exclusively Activated by Designer Drugs (DREADDs) Using Directed Molecular Evolution," Current Protocols in Neuroscience, 50(1), 4.33.1-4.33.25, 2010.

[44] D. S. Wilson and A. D. Keefe, "Random mutagenesis by PCR," Current Protocols in Molecular Biology, 51(1), 8.3.1-8.3.9, 2000.

[45] V. Nawaratne, K. Leach, N. Suratman, R. E. Loiacono, C. C. Felder, B. N. Armbruster et al, "New Insights into the Function of M4 Muscarinic Acetylcholine Receptors Gained Using a Novel Allosteric Modulator and a DREADD (Designer Receptor Exclusively Activated by a Designer Drug)," Molecular Pharmacology, 74(4), 1119-1131, 2008.

[46] C. Lüscher and P. A. Slesinger, "Emerging roles for G protein-gated inwardly rectifying potassium (GIRK) channels in health and disease," Nature Reviews Neuroscience, 11(5), 301-315, 2010.

[47] M. E. Tipps and K. J. Buck, "Girk Channels: A Potential Link Between Learning and Addiction," 123, 239-277, 2015.

[48] D. Atasoy, J. N. Betley, H. H. Su, and S. M. Sternson, "Deconstruction of a neural circuit for hunger," Nature, 488(7410), 172-177, 2012.

[49] B. R. Conklin, E. C. Hsiao, S. Claeysen, A. Dumuis, S. Srinivasan, J. R. Forsayeth et al, "Engineering GPCR signaling pathways with RASSLs," Nature Methods, 5(8), 673-678, 2008.

[50] G. M. Alexander, S. C. Rogan, A. I. Abbas, B. N. Armbruster, Y. Pei, J. A. Allen et al, "Remote Control of Neuronal Activity in Transgenic Mice Expressing Evolved G protein-couple receptors," Neuron, 63(1), 27-39, 2009.

[51] C. Agulhon, K. M. Boyt, A. X. Xie, F. Friocourt, B. L. Roth, and K. D. McCarthy, "Modulation of the autonomic nervous system and behaviour by acute glial cell Gq protein-coupled receptor activation in vivo," The Journal of Physiology, 591(22), 5599-5609, 2013. 
[52] M. S. Farrell and B. L. Roth, "Pharmacosynthetics: Reimagining the pharmacogenetic approach," Brain Research, 1511, 6-20, 2013.

[53] T. J. Ahonen, M. Rinne, P. Grutschreiber, K. Mätlik, M. Airavaara, D. Schaarschmidt et al, "Synthesis of 7B-hydroxy-8-ketone opioid derivatives with antagonist activity at mu- and delta-opioid receptors," European Journal of Medicinal Chemistry, 151, 495-507, 2018.

[54] P. Kunz, T. Flock, N. Soler, M. Zaiss, C. Vincke, Y. Sterckx et al, "Exploiting sequence and stability information for directing nanobody stability engineering," Biochimica et Biophysica Acta - General Subjects, 1861(9), 2196-2205, 2017.

[55] M. R. Bruchas and B. L. Roth, "New Technologies for Elucidating Opioid Receptor Function," Trends in Pharmacological Sciences, 37(4), 279-289, 2016.

[56] E. Vardy, J. E. Robinson, C. Li, R. H. J. Olsen, J. F. DiBerto, P. M. Giguere et al, "A New DREADD Facilitates the Multiplexed Chemogenetic Interrogation of Behavior," Neuron, 86(4), 936-946, 2015. 